\begin{tabular}{|l|c|c|c|}
\hline Eiszeitalter und Gegenwart & $\mathbf{5 5}$ & $\begin{array}{c}25-42 \\
4 \text { Abb., } 1 \text { Tab. }\end{array}$ & Hannover 2005 \\
\hline
\end{tabular}

\title{
Zur Stratigraphie des Saale-Glazials in Niedersachsen und zu Korrelationsversuchen mit Nachbargebieten
}

\author{
Klaus-Dieter Meyer ${ }^{*}$
}

Keywords: Middle Pleistocene, Saalian, Drentheand Warthe-Stadial, tills, outwash, indicator boulders, Lower Saxony.

Kurzfassung: Von verschiedener Seite unternommene Korrelations-Versuche zum Saale-Komplex in Norddeutschland sind der Anlass, erneut die Stratigraphie dieses Zeitraums aus niedersächsischer Sicht darzustellen.

Da das Holstein-Interglazial der Typus-Bohrung Bossel kürzlich mit der Th/U- Methode in Tiefsee-Stufe 9 datiert wurde, kommen für die jüngeren Thermomere Schöningen und Reinsdorf die Abschnitte 9a bis 7 in Frage. Die Speere aus dem Reinsdorf sind daher auf rund 270000 - 240000 Jahre zu veranschlagen.

Die durch drei Eisvorstöße gegliederte saaleeiszeitliche Glaziärfolge wird kurz charakterisiert. Bisher unpublizierte Leitgeschiebezählungen aus SW-Niedersachsen werden vorgestellt; zusammen mit Aufschlussuntersuchungen und Kartierungen lassen sich daraus keine weiteren Unterteilungen des Haupt-Drenthe-Stadiums ableiten.

Der zweite Saale-Vorstoß („Jüngere Drenthe“) sollte aus stratigraphischen und petrographischen Gründen nicht zum Warthe-Stadium gestellt werden. Letzteres ist durch ein ostbaltisches Geschiebespektrum charakterisiert und mit den jeweils jüngsten saalezeitlichen Vorstößen der Nachbarländer zu korrelieren. Warmzeitliche organogene Sedimente zwischen den Eisvorstößen fehlen und sind aufgrund der geologischen Situation unwahrscheinlich.

\footnotetext{
*) Anschrift des Verfassers: Prof. Dr. K.-D. Meyer, Engenser Weg 5, D-30938 Burgwedel-Oldhorst
}

[On the Stratigraphy of the Saalian in Lower Saxony and its Correlation with Adjacent Areas]

Abstract: The Saalian, which directly follows the Holsteinian, is a complex stratigraphic unit. Recently the Holsteinian interglacial was correlated on the basis of Th/U dates with MIS 9. Therefore, the Reinsdorf interglacial from the Schöningen opencast mine, where the famous spears were found, has to be younger than 400000 years, probably c. 270000 (correlated with MIS 9a) or c. 240000 years (correlated with MIS 7).

These warm periods clearly took place before the first Saalian advance, i.e. before the main Drenthian advance. This advance, which reached the Netherlands, left a till containing erratics mostly from southern and central Sweden. In SW Lower Saxony, this till is directly overlain by brown-red till of East Baltic character and is interpreted as a later deposit before the ice retreated.

The Younger Drenthian advance did not cross the Weser and Aller rivers and left a till containing more Upper Cretaceous material (flint and chalk) than the main Drenthian till. This advance, which corresponds to the Lamstedt Phase, should not be assigned to the Warthian. The Warthian till, which can clearly be distinguished by its East Baltic character, is correlated with the youngest Saalian till in the adjacent areas.

The ice-free periods between the individual ice advances of the Saalian glaciation were probably short. Numerous depressions in the land surface of all the different tills, mostly dead-ice hollows or ice-tongue basins, are only infilled with Eemian and Weichselian sediments. It can be concluded that no interglacials existed between the main Drenthian advance and the Eemian. 


\section{Einleitung}

Das gesamte niedersächsische Flachland war saalezeitlich vergletschert, selbst in das Bergland drang das Eis hinein, den Flusstälern folgend; hierbei kam es zur Verzahnung glaziärer und fluviatiler Sedimente. Im Nordsee-Küstengebiet liefern marine Interglazial-Sedimente obere und untere Zeitmarken des "Saale-Komplexes“. Als einziges Bundesland liegt somit in Niedersachsen eine Verknüpfung glaziärer sowohl mit marin-interglazialen wie mit wichtigen Terrassen-Sedimenten vor. Über die benachbarten Niederlande und Nordrhein-Westfalen besteht eine Brückenfunktion zum Rheingebiet und damit indirekt zur alpinen Vereisung. Korrelationsversuche sind daher angebracht, auch in jüngerer Zeit mangelte es daran nicht. Trotz der ständig verbesserten Kenntnislage (vielleicht aber auch wegen der Datenfülle) ist es für den Einzelnen jedoch nicht einfach, einen angemessenen Überblick über die Verhältnisse im Nachbarland zu behalten, und so ist es kein Wunder, wenn sich Missdeutungen einschleichen. Im vorliegenden Beitrag soll auf einige solcher Fehldeutungen eingegangen werden, wobei jeweils eine kurze Betrachtung der einzelnen stratigraphischen Einheiten des "Saale - Komplexes" in Niedersachsen vorausgeschickt wird. Dies ist auch deshalb angebracht, weil seit der letzten derartigen Übersicht (BENDA 1995) einige neuere meist unpublizierte Erkenntnisse vorliegen.

\section{Unterer Saale-Komplex}

In Niedersachsen wird die Abgrenzung der saalezeitlichen gegenüber den elsterzeitlichen Ablagerungen durch limnische und marine Vorkommen des Holstein-Interglazials ermöglicht; die Grenze liegt am Übergang der borealen zur subarktischen Phase, d. h. die darüber liegenden kalt- und warmzeitlichen Abschnitte (Fuhne-Kaltzeit, Dömnitz bzw. Wacken-Warmzeit) etc. werden bereits zum "Saale-Komplex“ gerechnet (LitT \& Turner 1993). Da bislang eine verlässliche Korrelierung mit der TiefseeChronologie nicht möglich bzw. umstritten ist, sind darauf basierende absolute Altersangaben mit Vorsicht zu betrachten. Nunmehr legen GeyH \& Müller 2005 neue Th/U-Daten hauptsächlich aus der Typus-Lokalität-Bohrung Bossel bei Stade vor (Müller \& Höfle 1994), die eine Korrelation des Holstein mit der Tiefsee-Stufe 9 belegen, wie es u.a. in den Niederlanden schon bisher zumeist angenommen wurde (u.a. ZAGWIJN 1996).

Damit erhebt sich erneut die Frage nach der Position bzw. dem absoluten Alter der Warmzeiten innerhalb des Unteren Saale-Komplex, d.h. ob Dömnitz/Wacken und die damit zumindest teilweise korrespondierenden Warmzeiten aus dem Tagebau Schöningen (Reinsdorf- und Schöningen-Warmzeit, URBAN 1995) zur Gänze oder teilweise zur Tiefseestufe $7 \mathrm{zu}$ stellen sind bzw. teilweise noch in einen jüngeren $\mathrm{Ab}$ schnitt der Tiefseestufe 9 (9c oder 9a). Welcher der beiden man auch immer den Vorzug gibt - die kaltzeitlichen Sedimente zwischen den Stufen 7 und 9 sind sowohl was Mächtigkeit und Verbreitung anbelangt so unbedeutend, dass man sich schwer darunter die Präsenz einer ganzen „Eiszeit“ vorstellen kann.

Die Altersfrage ist auch aus archäologischer Hinsicht höchst interessant. Die berühmten Holzspeere der Reinsdorf-Warmzeit des Tagebaus Schöningen sind nach Thieme (zuletzt 1999) 400000 Jahre alt. Kürzlich haben sich JÖris \& BAALES (2003) mit dieser Frage beschäftigt und kommen zu der Einstufung des Reinsdorf in Stufe 9e mit „nur“ noch ca. 310.000 Jahren.

Bei dieser Einstufung wird noch von der Existenz einer Drenthe/Warthe-Warmzeit ausgegangen. Da dies aus noch zu diskutierenden Gründen verworfen werden muss, ist von einer 
theoretischen Position des Reinsdorf in Stufe 9 a oder $7 \mathrm{e}$ auszugehen und damit ein absolutes Alter der Speere von ca. 270000 oder 240000 Jahren. Weitere Untersuchungen, nicht zuletzt absolute Altersdatierungen der betreffenden Interglaziale bleiben abzuwarten, auch wäre eine Vorlage der geologischen Detailprofile von Schöningen wünschenswert.

Die Richtigkeit dieser Einstufungen vorausgesetzt, bleibt für den gesamten glaziären $\mathrm{Ab}$ schnitt des Saale-Komplexes, d. h. für Drentheund Warthe-Stadium, nur eine Korrelation mit Tiefsee-Stufe 6 und damit der Zeitraum von rund 200000 bis 130000 Jahren.

\section{Oberer Saale-Komplex}

Dieser Abschnitt umfasst den Bereich vom Ende der Dömnitz/Wacken-Warmzeit bis zum Beginn des Eem (Litt \& Turner 1993). Er wird in Niedersachsen in Drenthe- und Warthe-Stadium gegliedert, ungeachtet der Tatsache, dass keine interstadialen Ablagerungen dazwischen nachgewiesen wurden. Das Drenthe-Stadium wird wiederum in einen älteren und jüngeren Abschnitt (Haupt-Drenthe und Jüngere Drenthe) geteilt. Bei der lithostratigraphischen Untergliederung erwiesen sich v.a. die Grundmoränen als brauchbare Leithorizonte, charakterisiert durch Unterschiede im Leitgeschiebeinhalt, z.T. auch durch Feinkies- und Schwermineralspektren, Farbe, Korngröße und Kalkgehalt. Eingeschränkt wird die stratigraphische Verwertbarkeit der lithologischen Parameter durch fazielle Wiederholungen in der Abfolge.

\section{1 Älteres Drenthe-Stadium}

Der Saale-Hauptvorstoß (Hamelner Phase des Drenthe-Stadiums) hinterließ außer gla- zilimnischen und glazifluviatilen Sedimenten eine mächtige Grundmoräne mit süd- bis mittelschwedischer Geschiebevormacht bei relativ einheitlicher Zusammensetzung (Drenthe-Hauptmoräne). Ihr ist im westlichen Niedersachsen bis in die Niederlande lokal eine ostbaltisch geprägte braunrote Grundmoräne aufgesetzt, die in Niedersachsen als späterer Nachschub des gleichen Eisvorstoßes gedeutet wird, ohne dass das Gebiet vorher eisfrei wurde. Gerade dies wurde aber in jüngerer Zeit wieder in Frage gestellt und in der roten Moräne das Produkt eines eigenen Vorstoßes gesehen (Nowel 2003, Skupin, Speetzen \& ZandsTRA 2003). Die letzteren Autoren rechnen in Nordrhein-Westfalen sogar mit drei einzelnen, mehr oder weniger kurz aufeinander folgenden Vorstößen innerhalb des Haupt-Drenthe-Stadiums, wobei es zwischen dem ersten und zweiten Vorstoß wohl nur eine kurze Stagnationszeit gegeben habe.

Dem zweiten Vorstoß wird eine Geschiebegemeinschaft mit relativ hohem Anteil an Dalarna-Geschieben zugeschrieben. Geschiebekollektionen mit relativ hohen Anteilen solcher Gesteine sind in Niedersachsen schon in den Vorschüttsedimenten durchaus nicht ungewöhnlich. Teils stammen sie aus umgelagerten elsterzeitlichen Sedimenten wie in Ostfriesland (Meyer 1970), wie aus der Beimengung norwegischer Leitgeschiebe hervorgeht, teils kann dies Phänomen als innerhalb der normalen Schwankungsbreite gelegen aufgefasst werden.

Von den auf Abbildung 1 und Tabelle 1 dargestellten Leitgeschiebezählungen aus SW-Niedersachsen liegt der Anteil der Dala-Gesteine der Proben 3 und 7, die in Abbildung 1 am weitesten im NW-Sektor liegen, bei 23 bzw. $27 \%$. Da die Proben 15 bzw. 10 \% norwegische Leitgeschiebe enthalten, liegt eine Aufarbeitung von elsterzeitlichem Material nahe. In Probe 8 aber, die mit $21 \%$ ebenfalls hohe Dala-Werte hat, fehlt jedoch norwegisches Material. In Probe 9, die wieder relativ weit im NW des Diagramms 


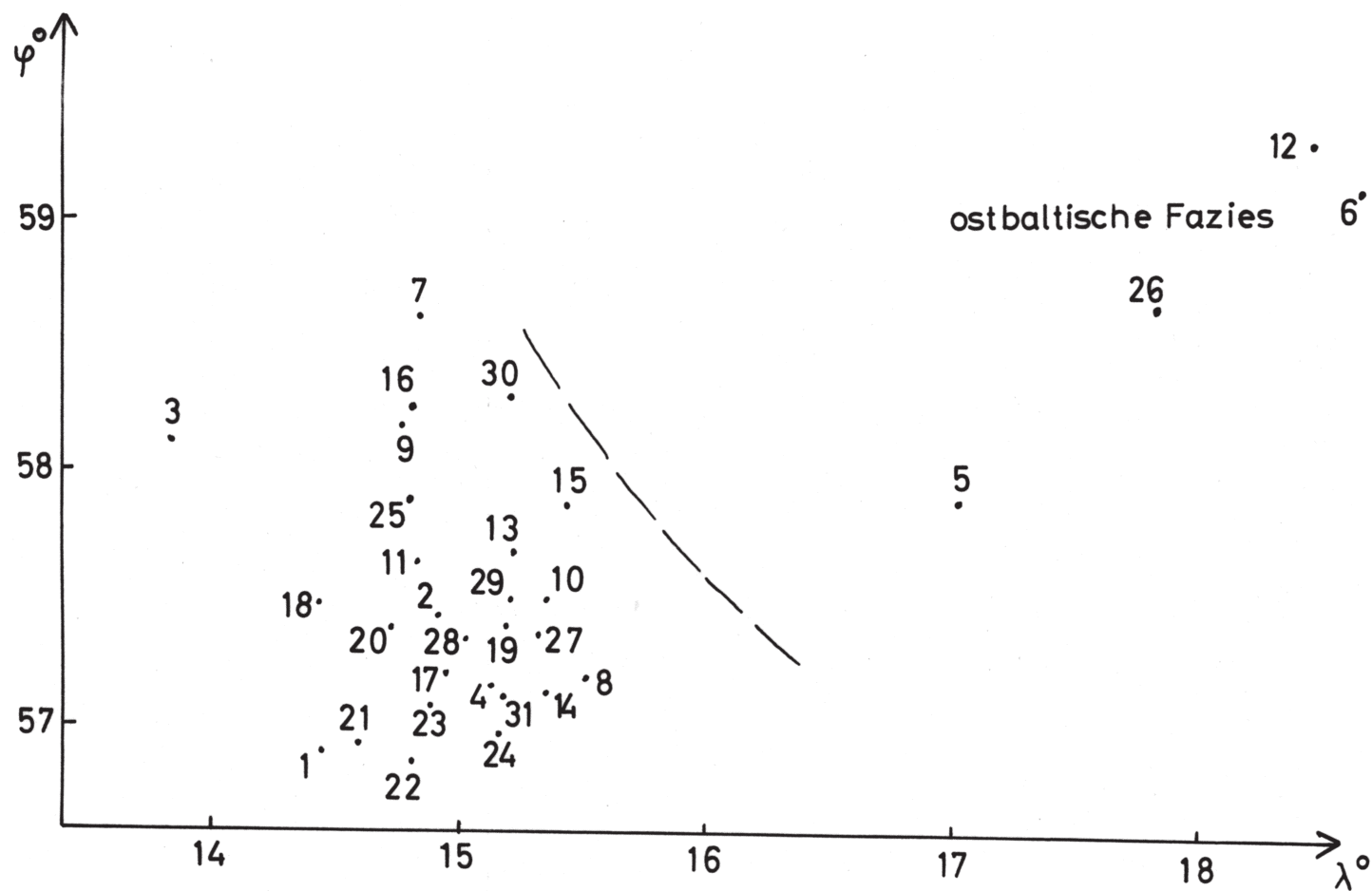

Abb.1: Theoretische Geschiebezentren (TGZ) von Leitgeschiebezählungen drenthezeitlicher Glaziärsedimente (Grundmoränen, Schmelzwasserkies) aus Südwest-Niedersachsen. Fundpunkte s. Tab.1.

Fig. 1: Theoretical home center of erratics from tills and outwash in SW Lower Saxony.

liegt, treten mit $29 \%$ sogar die höchsten DalaWerte auf, obwohl nur ein fragliches Oslo-Gestein gefunden wurde. Die Proben 10 und 11, die unweit voneinander im Diagramm liegen, wurden nur in $2 \mathrm{~m}$ Vertikalabstand entnommen. Der Dala-Anteil der oberen Probe liegt bei $12 \%$, in der unteren aber bei $22 \%$.

Diese beiden Proben (Nr. 10 und 11) stammen aus der SW-Wand der kleinen Sandgrube am Flintenberg bei Rathlosen. Bemerkenswert ist der Anteil von paläozoischem Kalkstein (10 \%) in der unteren Probe, während die Schmelzwasserablagerungen westlich der Weser ansonsten meist vollständig entkalkt sind. Kalkhaltige Kiese $(11 \%)$ fanden sich sonst noch in gestauchter Lagerung in der Sandgrube $2 \mathrm{~km}$ südwestlich Estringen, (Probe 28). Auffällig ist ferner noch die Position des Flintenberges an der Westseite eines Tales, erinnernd an die von K. Richter (1950) beschriebenen Oser der Syker Geest. Aus Os-verdächtiger Position stammen auch die Proben 20 und 21 aus der Sandgrube in den Harberger Fichtenkämpen auf Blatt 3320 Liebenau (LüTtig \& MeYer 1980: 102 ), dort allerdings kalkfrei.

Die Anteile an Dalagesteinen sind also schon im Vorschüttkörper der Haupt-Drenthe beträchtlichen Schwankungen unterworfen; unterschiedliche Eisvorstöße lassen sich damit nicht begründen. Natürlich soll damit eine mögliche Aufspaltung des Drenthe-Eises im Randbereich nicht grundsätzlich in Abrede gestellt werden, nur sollten geschiebekundliche Differenzierungen auch durch Überlagerungsfälle belegt sein. Zwischen dem zweiten und dritten Vorstoß soll es nach Skupin, Speetzen \& Zandstra (1993, 
Tab. 1: Leitgeschiebezählungen drenthezeitlicher Glaziärsedimente aus SW-Niedersachsen, Fraktion 2-6cm

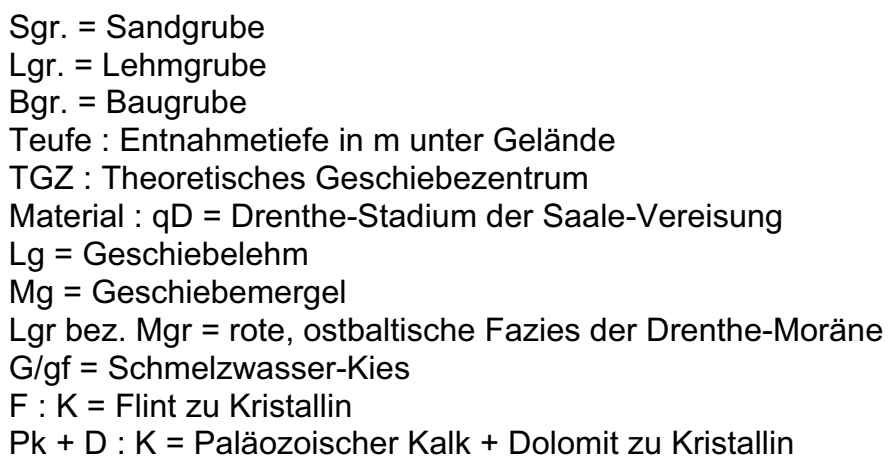

\begin{tabular}{|c|c|c|c|c|c|c|c|c|c|}
\hline $\mathrm{Nr}$ & Fundort & TK 25 & R-Wert & H-Wert & Teufe & TGZ & Material & $F: K$ & $\begin{array}{c}\text { PK + D : } \\
K\end{array}$ \\
\hline 1 & NE Börger, Sgr. & 3011 & 3403800 & 5869000 & 10 & $14,45-56,91$ & $\mathrm{qD} / \mathrm{G} / \mathrm{gf}$ & 1,5 & \\
\hline 2 & E Raken, Sgr. & 3109 & 2585860 & 5852740 & 3 & $14,91-57,44$ & $\mathrm{qD} / / \mathrm{Lg}$ & 1,04 & \\
\hline 3 & N Werpeloh, Sgr. & 3111 & 3400100 & 5862600 & 4 & $13,86-58,12$ & $\mathrm{qD} / \mathrm{G} / \mathrm{gf}$ & 0,85 & \\
\hline 4 & SW Cloppenburg, Lgr. & 3114 & 3432760 & 5855000 & $5-7$ & $15,13-57,17$ & $\mathrm{qD} / / \mathrm{Mg}$ & 0,82 & 0,46 \\
\hline 5 & $\begin{array}{l}\text { W Schneiderkrug, } \\
\text { Bgr. }\end{array}$ & 3115 & 3448200 & 5853480 & $1,5-2,5$ & $17,03-57,91$ & $\mathrm{qD} / / \mathrm{Mgr}$ & 0,02 & 4 \\
\hline 6 & NNW Barlage, Lgr. & 3213 & 3428160 & 5847440 & 0 & $18,76-59,18$ & $\mathrm{qD} / \mathrm{Mgr}$ & 0,01 & 2,73 \\
\hline 7 & SW Krüllenberg, Sgr. & 3218 & 3487640 & 5843480 & 6 & $14,84-58,62$ & $\mathrm{qD} / \mathrm{G} / \mathrm{gf}$ & 0,65 & \\
\hline 8 & E Wesenstedt, Sgr. & 3218 & 3482640 & 5846200 & $1,2-1,4$ & $15,51-57,19$ & $\mathrm{qD} / \mathrm{G} / \mathrm{gf}$ & 1,53 & \\
\hline 9 & Vohrde, Sgr. & 3218 & 3482640 & 5843800 & 2 & $14,77-58,18$ & $\mathrm{qD} / \mathrm{G} / \mathrm{gf}$ & 1,49 & \\
\hline 10 & E Rathlosen, Sgr. & 3218 & 3483000 & 5841180 & $1-1,5$ & $15,35-57,50$ & qD/G/gf & 0,65 & \\
\hline 11 & E Rathlosen, Sgr. & 3218 & 3483000 & 5841180 & 4 & $14,83-57,65$ & qD/G/gf & 1,11 & 0,31 \\
\hline 12 & S Herzlake, Lgr. & 3311 & 3405600 & 5837160 & $1,5-3$ & $18,46-59,36$ & $\mathrm{qD} / / \mathrm{Lgr}$ & 0,04 & \\
\hline 13 & E Dickel, Bgr. & 3316 & 3465650 & 5834425 & 1 & $14,22-57,69$ & $\mathrm{qD} / / \mathrm{Lg}$ & 1 & \\
\hline 14 & S Jacobidrebber, Sgr. & 3316 & 3460950 & 5834900 & 2 & $15,35-57,15$ & $\mathrm{qD} / / \mathrm{Lg}$ & 0,98 & \\
\hline 15 & W Sulingen, Sgr. & 3318 & 3484540 & 5838700 & $1-1,5$ & $15,43-57,87$ & qD/G/gf & 1,57 & \\
\hline 16 & SSE Barenburg, Sgr. & 3318 & 3487100 & 5830000 & $1-2$ & $14,81-58,26$ & $\mathrm{qD} / \mathrm{G} / \mathrm{gf}$ & 0,64 & \\
\hline 17 & SE KI. Lessen, Lgr. & 3318 & 3484820 & 5834520 & $2-3$ & $14,94-57,22$ & $\mathrm{qD} / / \mathrm{Lg}$ & 0,41 & \\
\hline 18 & W Lemke, Sgr. & 3320 & 3508000 & 5835410 & $2-4$ & $14,44-57,49$ & $\mathrm{qD} / / \mathrm{Lg}$ & 1,19 & \\
\hline 19 & Harbergen, Sgr. & 3320 & 3500480 & 5839580 & $7-9$ & $15,19-57,40$ & $\mathrm{qD} / / \mathrm{Mg}$ & 1,21 & 0,31 \\
\hline 20 & Harbergen, Sgr. & 3320 & 3500480 & 5839580 & 4,5 & $14,73-57,49$ & $q D / G / g f$ & 0,98 & \\
\hline 21 & Harbergen, Sgr. & 3320 & 3500480 & 5839580 & 1,5 & $14,60-56,94$ & $q D / G / g f$ & 0,69 & \\
\hline 22 & SW Neuenwalde, Sgr. & 3314 & 3442040 & 5819440 & $2-5$ & $14,81-56,86$ & $\mathrm{qD} / / \mathrm{Lg}$ & 0,87 & \\
\hline 23 & E Oldorf, Sgr. & 3415 & 3450400 & 5822600 & $0,5-5$ & $14,89-57,11$ & $\mathrm{qD} / / \mathrm{Lg}$ & 0.82 & \\
\hline 24 & NW Kellenberg, Bgr. & 3417 & 3466200 & 5829350 & 2 & $15,16-56,97$ & $\mathrm{qD} / / \mathrm{Lg}$ & 1,44 & \\
\hline 25 & W Kirchdorf, Bgr. & 3418 & 3487650 & 5829270 & $2-3$ & $14,80-57,89$ & $\mathrm{qD} / / \mathrm{Lg}$ & 0,87 & \\
\hline 26 & S Lingen, Bgr. & 3509 & 2589700 & 5816200 & $5-6$ & $17,82-58,70$ & $\mathrm{qD} / / \mathrm{Mgr}$ & 0,12 & 3,9 \\
\hline 27 & S Lingen, Bgr. & 3509 & 2589700 & 5816200 & $7-8$ & $15,32-57,36$ & $\mathrm{qD} / / \mathrm{Mg}$ & 1,05 & 0,51 \\
\hline 28 & NW Estringen, Sgr. & 3510 & 2590980 & 5817950 & 6 & $15,03-57,35$ & qD/G/gf & 0,66 & 0,26 \\
\hline 29 & S Diepenau, Lgr. & 3518 & 3480500 & 5808455 & 3 & $15,22-57,50$ & $q D / G / g f$ & 1,17 & \\
\hline 30 & Steide, Sgr. & 3609 & 2590230 & 5797810 & $1,5-2,5$ & $15,21-58,30$ & $\mathrm{qD} / \mathrm{G} / \mathrm{gf}$ & 0,84 & \\
\hline 31 & Staelberg, Lgr. & 3609 & 2585120 & 5805360 & $1-4$ & $15,18-57,12$ & $\mathrm{qD} / / \mathrm{Lg}$ & 0,76 & \\
\hline
\end{tabular}


2003) zu einem regionalen Abschmelzen gekommen sein. Auf diese Aussage beruft sich auch Nowel (2003), der eine Korrelation der roten Drenthe-Moräne mit der S II-Moräne in Brandenburg („Fläming-Glazial“, Cерек 1967) vorschlägt. Mit den Befunden in Niedersachsen ist dies unvereinbar, weshalb hier etwas näher darauf eingegangen werden soll.

Die rote Grundmoräne unterscheidet sich sehr deutlich von der liegenden Drenthe-Hauptmoräne. Sie ist, wie seit langem bekannt, durch eine ostbaltische Geschiebegemeinschaft gekennzeichnet, v. a. viel Åland-Kristallin, Roten Ostsee-Quarzporphyr etc., die über 50 $\%$ des Leitgeschiebeanteils erreichen können. Entsprechend liegen die TGZ weit im NE des Diagramms (Abb. 1, Pr. 5, 6, 12 und 26). Im Bereich der Proben 6 und 12 liegen auch die TGZ vom „Roten Geschiebelehm der Niederlande“ (LÜTTIG \& MAARLEVELLD 1961).

Die ostbaltische Herkunft wird ferner durch den hohen Gehalt an Dolomit-Geschieben (bis $15 \%$ ) und den sehr hohen Anteil an grauen paläozoischen Kalksteinen (bis $60 \%$ ) unterstrichen, darunter viel ordovizischer sog. Ostseekalk (bis $8 \%$ ). Zusammen können die Karbonatgesteine rund $75 \%$ des Gesamtbestandes erreichen. Auffällig ist, dass die roten ordovizischen Orthocerenkalke von Öland äußerst selten sind, d.h. der Ostseegletscher hat aus diesem Bereich kaum Material aufgenommen. Old Red-Sandsteine aus dem baltischen Devon, darunter nicht selten Kugelsandstein, können einige Prozent erreichen und sogar anteilsmäßig den Feuerstein übertreffen, der in manchen Proben auch fast fehlt.

Die für eine Geschiebezählung nötige Anzahl von Leitgeschieben zu sammeln ist wegen des hohen Karbonat-Anteils sehr aufwändig. Die vorhergehend geschilderte charakteristische Zusammensetzung spiegelt sich aber auch im Feinkiesspektrum wider; sogar im Handbohrer ist die rote Moräne zumindest im nicht-entkalkten Zustand zu erkennen, zumal bei hohem
Tongehalt, der bis auf $70 \%$ ansteigen kann und dann eher an Beckensedimente erinnert. Hoher Ton- und Karbonatgehalt sind die Ursache, weshalb die Moräne manchmal schon in gut $1 \mathrm{~m}$ unter Gelände noch als Geschiebemergel auftritt - ungewöhnlich für das Altmoränengebiet, wo normalerweise die gesamte HauptDrenthemoräne völlig entkalkt ist, auch bei Mächtigkeiten um $10 \mathrm{~m}$.

Es gibt aber auch tonärmere, sandige und dann entkalkte Varianten der ostbaltischen Moräne. Eine solche wird in den Niederlanden als „Emmen-Typ" bezeichnet (Zandstra 1976) und dem tonreicheren „Voorst-Typ“ gegenübergestellt. Es zeigt sich jedoch, dass bei größerer Mächtigkeit (2,5 m ) wie in der Baugrube des KKW Lingen (Pr. 26, Tab.1 und Abb.1) diese sandige Moräne (nur 11,5\% Ton) mit $58 \%$ paläozoischem Kalkstein und $15 \%$ Dolomit bei nur 2\% Flint die gleiche Geschiebezusammensetzung hat wie die tonreiche Fazies und deshalb nicht einem gesonderten Typ zugeordnet werden kann.

Dieser etwa $6 \mathrm{~km}$ südlich der Stadt Lingen gelegene Aufschluss (Abb. 2) war noch insofern bemerkenswert, als hier die rote ostbaltische Moräne direkt, d.h. ohne irgendeine Trennschicht, einen über $4 \mathrm{~m}$ mächtigen grauen, sandigen Geschiebemergel überlagert, der ein typisch süd- bis mittelschwedisches Leitgeschiebespektrum aufweist (Pr. 27, Tab.1 und Abb.1). Die Orientierung der Geschiebe-Längsachsen dieser grauen Grundmoräne weist ein NW-SE gerichtetes Maximum auf, d.h. etwas spitzwinklig zu dem unweit westlich gelegenen Embürener Stauchrücken bzw. dessen nördlicher Fortsetzung. Derartig NW-SE orientierte Gebiete sind im westlichen Niedersachsen noch an anderen Stellen, z.B. in der Syker Geest, anzutreffen. Sie sind eher auf Differenzialbewegungen im Inlandeis als auf eigenständige Gletschervorstöße zurückzuführen. Die hangende rote Moräne wies keine bevorzugte Orientierung auf. Ebenfalls relativ geringe Tongehalte (18,7 und 
$19,4 \%)$ zeigte die rote Moräne in der ehemaligen Ziegelei Felsen bei Herzlake (TK $25 \mathrm{Nr}$. 3311; Farbabbildung 17 bei MeYer 2002); mit $4 \mathrm{~m}$ max. Mächtigkeit erbohrt. Auch hier wird grauer Geschiebemergel in mehr als $10 \mathrm{~m}$ Stärke direkt überlagert. Der Tongehalt der roten Moräne von Felsen liegt nur wenig niedriger als im „Emmen-Typ“ von Emmerschans (ca. $24 \%$, ZandSTRA 1976). Aus niedersächsischer Sicht scheint es nicht überzeugend, aus diesem relativ tonarmen Vorkommen eine jüngere ostbaltische Phase ableiten zu wollen, die mit NNW-SSE-Richtung über die Nordsee gekommen sein soll (Rappol et al. 1989, KuUiving et al. 1991).

Besonders die Vorkommen mit hohem Ton- gehalt dienten bis in die 70er Jahre als ZiegelRohstoff. Nachteilig war dabei der oft bei 1 - 2 $m$ unter Gelände einsetzende hohe Karbonatgehalt und andererseits das schnelle Auskeilen, weshalb dann, wie in der Ziegelei Moormann bei Cloppenburg, auch die liegende sandige Drenthe-Hauptmoräne mit abgebaut wurde (Pr. 4, Tab.1 und Abb.1), obwohl letztere mit nur 16,1 bzw. $21 \%$ erheblich weniger Ton enthielt als die dortige hangende rote Moräne mit 41,9 und 59,3\%. Untersuchungen der Tonfraktion von ostbaltischen Moränen in den Niederlanden (Haldorsen et al. 1989) ergaben geringen oder fehlenden Smektit-, aber hohen Illit-Gehalt, was ebenfalls auf ostbaltische Herkunft deutet. Außer in Ziegeleigruben und in vielen Boh-

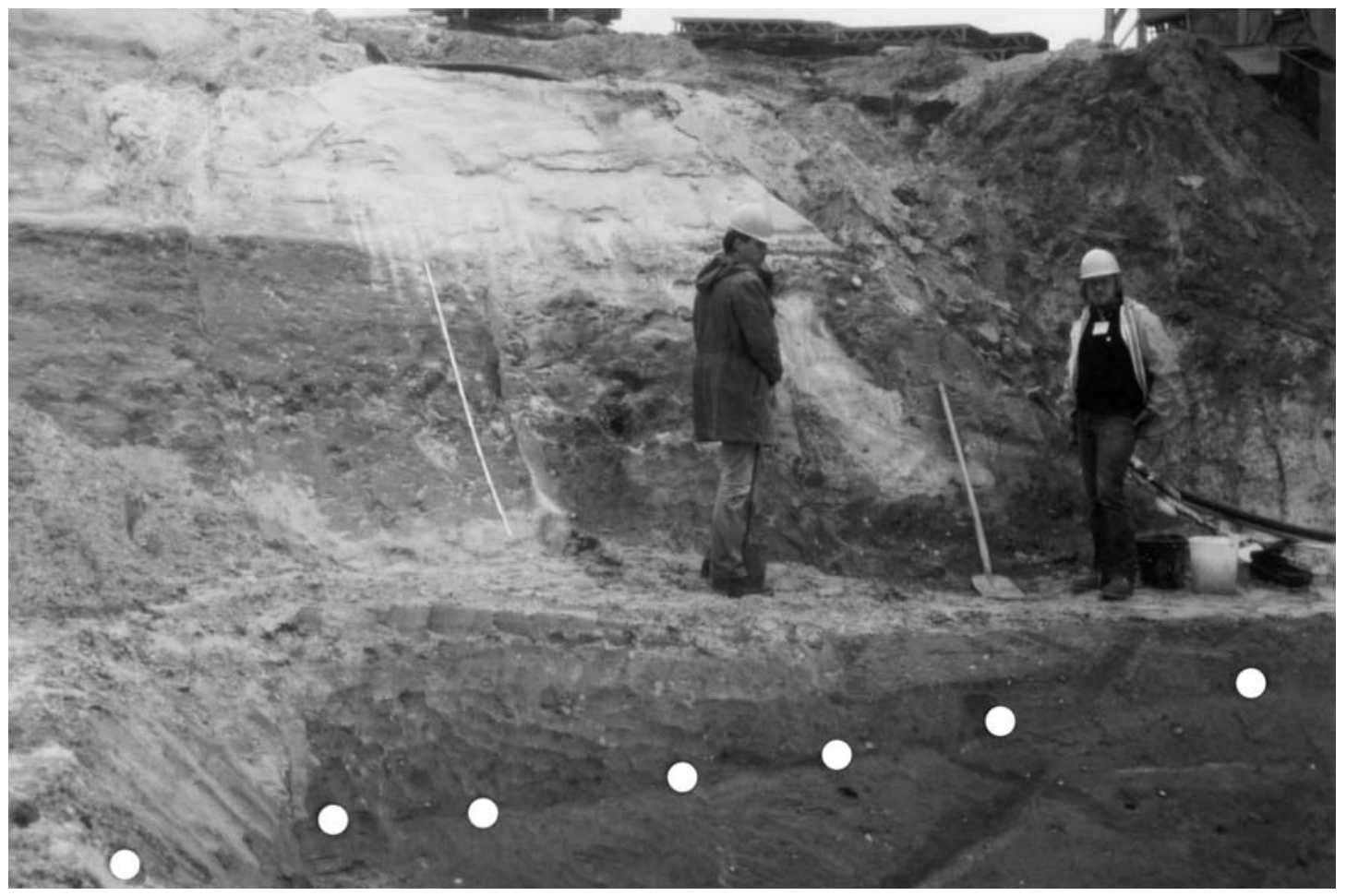

Abb. 2: Braunroter, toniger Geschiebemergel (Pr. 26, Tab.1 und Abb.1) (= ostbaltische Fazies der DrentheHauptmoräne) direkt auf grauem, sandigen Geschiebemergel (Probe 27, Tab.1 und Abb.1), überlagert von weichselzeitlichem fluviatilen Sand der Emsniederung; zuoberst Düne. TK 25 Lingen-Süd Nr. 3509. Baugrube KKW Lingen 1984.

Fig. 2: Brownish-red till (east baltic facies) above greyish, sandy till. Construction pit Lingen. 
rungen konnte die rote Moräne auch in Baugruben, besonders aber in km-langen Erdgas-, Wasserleitungs- und Entwässerungsgräben (Abb. 3) beobachtet werden. Dabei zeigte sich, dass sie keinesfalls als durchgehende Decke über der „normalen“ Moräne auftritt, sondern in der Regel nur auf wenige 100 oder $10 \mathrm{er} \mathrm{m}$, nicht selten auch nur auf wenige $m$ Länge $\mathrm{zu}$ verfolgen ist. Meist geschieht diese Überlagerung mit scharfer Grenze, gelegentlich mit gewisser Verzahnung. In keinem Fall waren Sandschichten oder eine periglaziale Steinsohle etc. zwischengelagert. Hätte es tatsächlich ein Eisrückschmelzen gegeben, so sollten in der nachfolgenden Periglazialzeit Steinpflaster oder Kryoturbationen etc. entstanden sein, die nicht überall beim Wiedervorstoß entfernt wären.
Auch sollten sich hin und wieder Vorschüttsande finden lassen.

Da alle diese Erscheinungen fehlen (auch in den Niederlanden), ist ein Eisrückzug höchst unwahrscheinlich. Beide so grundverschiedenen Moränen sind als verschiedene Fazies ein und derselben Vergletscherung zu deuten. Der rote Geschiebemergel, der in völlig gleicher Ausbildung auch in Polen und in baltischen Staaten auftritt (sowohl in weichselzeitlichen wie saalezeitlichen Abfolgen), kann aufgrund seiner $\mathrm{Zu}$ sammensetzung nicht als „normale“ Grundmoräne betrachtet werden. Wäre er über hunderte von $\mathrm{km}$ als Basalmoräne transportiert, müsste dabei mehr Fremdmaterial aufgenommen worden sein, v.a. Feuerstein. Vermutlich ist die rote Moräne inglazial transportiert, als eine Art

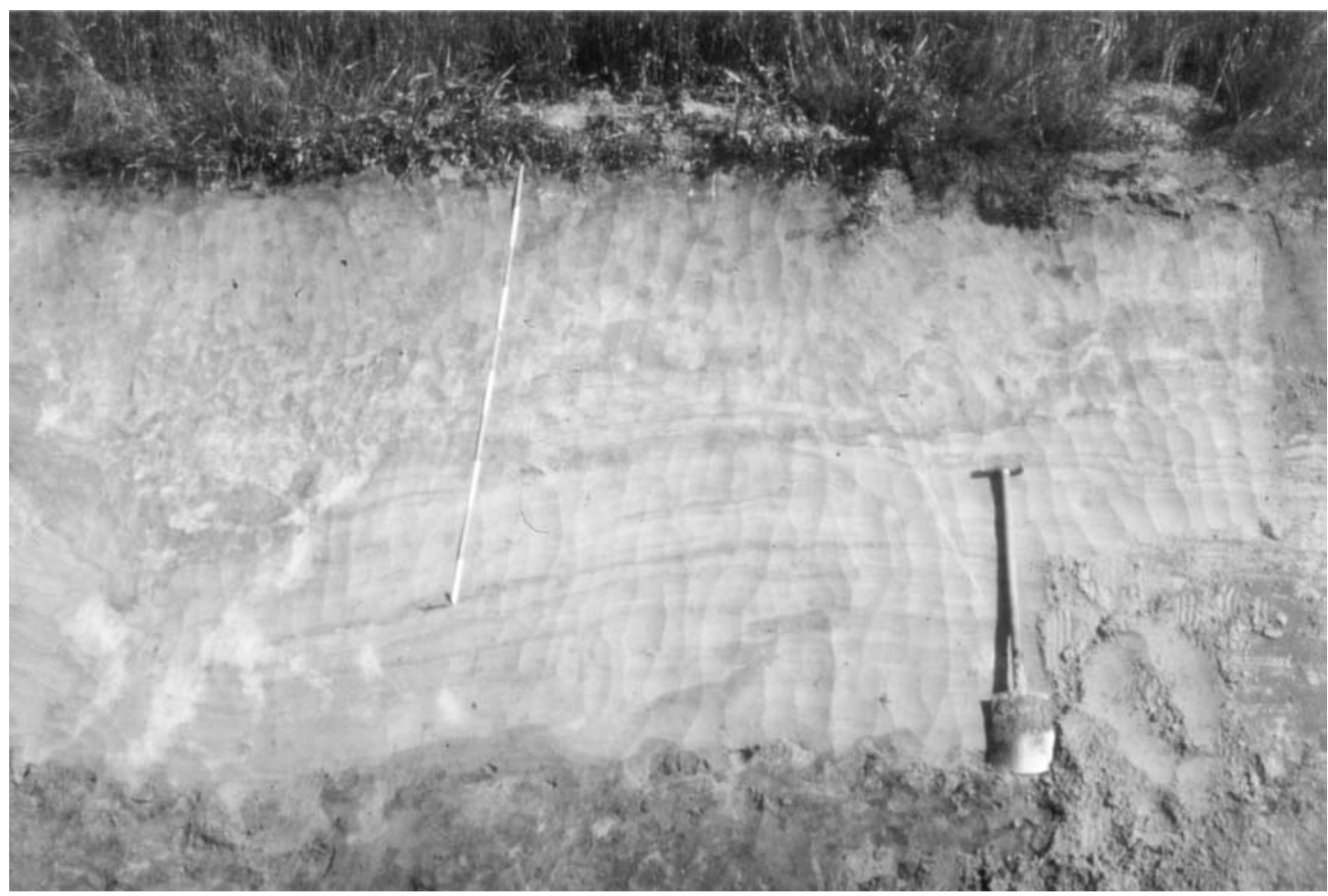

Abb. 3: Braunroter, stark toniger Geschiebelehm (= ostbaltische Fazies der Drenthe-Hauptmoräne) unter gebändertem weichselzeitlichen Flugdecksand; zuoberst Sandlöss. Entwässerungsgraben $1 \mathrm{~km}$ westlich Varenesch, TK 25 Goldenstedt Nr. 3216, Foto 1971.

Fig. 3: Brownish-red till (east baltic facies) below eolian coversand and sandy loess. Section Varenesch. 
„Nachschub“ gegen Ende des Drenthe-Hauptvorstoßes, vielleicht auch teilweise über Toteis hinweg. Dafür spricht auch die Verbreitung, die in Niedersachsen (von wenigen Ausnahmen abgesehen) auf das Gebiet der Nienburg-Meppener Geest beschränkt ist, genauer gesagt, auf das Gebiet westlich der Hunte. Weiter östlich, z.B. im Raum Hannover, ist nicht ein einziger Fund bekannt, trotz bester Aufschlussverhältnisse, tausender von Bohrungen und modernen geologischen Kartierungen. Es kann sich also nicht um eine Kenntnislücke handeln, was nicht ausschließt, dass man irgendwann einmal etwas findet.

Auch im Unterelbe-Gebiet ist trotz vieler, bis quadratkilometer-großer Aufschlüsse und massenhafter Bohrungen, in denen nicht selten die Drenthe-Hauptmoräne erschlossen wurde, auf niedersächsischer Seite nur zweimal rote Moräne am Top der grauen Moräne gefunden worden und das nur in dm-Stärke. Der eine Fund stammt aus der großen Sandgrube Eilendorf bei Buxtehude, in der seit Jahrzehnten die Drenthe-Hauptmoräne, in Schmelzwassersedimente eingeschaltet, aufgeschlossen ist. In ihrem Hangenden wurde ein dm-mächtiger, fast flintfreier roter Geschiebemergel gefunden (Meyer 1982: 43). Ähnlich war die Situation in der großen Sandgrube von Breetze bei Bleckede (MeYer 2004: 76).

Nördlich der Elbe beschrieb Ehlers (1992) aus Bohrungen in Hamburg-Langenhorn rote Moräne in einigen m Mächtigkeit mit Dolomit und hohem Kalksteingehalt im Hangenden der grauen Haupt-Drenthe-Moräne. EHLERs (1992) diskutiert ebenfalls inglazialen Transport und zieht zur Erklärung eine Verlagerung der Eisscheide heran. Auch aus Schleswig-Holstein (Dithmarschen) sind rotbraune Geschiebemergel mit ostbaltischer Prägung in gleicher stratigraphischer Position bekannt (STEPHAN 1980, 1998).

Allen diesen nordwestdeutschen Vorkommen der ostbaltischen Moräne im Hangenden der normalen sandigen Drenthe-Moräne ist ihr fleckenhaftes, äußerst lückenhaftes Vorkommen gemeinsam; Abbildung 4 zeigt schematisch diese Verhältnisse. Von einem durchgehenden Horizont wie auf der Abbildung 6 bei Nowel (2003: 66) kann keine Rede sein, ebenso wenig von einem vorherigen Rückschmelzen. Nicht akzeptabel ist auch die Annahme Nowels (2003: 59), die Stauchendmoränen der Rehburger Phase würden die Maximalausbildung eines „Saale 2-Vorstoßes“ markieren. Die Überfahrung dieser Stauchmoräne ist durch Spezialkartierungen, viele Bohrungen und zahlreiche Aufschlüsse eindeutig belegt, und zwar für sämtliche Teilabschnitte. Aus den Fürstenauerund Dammer Bergen, dem geradezu klassisch entwickelten Lobus der Rehburger Phase, liegen detaillierte strukturelle und sedimentologische Studien vor, die ebenfalls die Überfahrung belegen (VAN DER Wateren 1987). In den benachbarten Niederlanden ist dies gleichfalls erwiesen (VAN DEN BERg \& BeETs 1987).

Die in Abbildung 1 bei Nowel (2003: 48) dargestellte Verbreitung der roten Drenthe-Moräne im südwestlichen Niedersachsen erweckt den Eindruck flächenhaften Vorkommens im proximalen Bereich der Stauchendmoränen westlich von Quakenbrück; die rote Moräne tritt dort aber auf den meisten TK 25 überhaupt nicht auf. Bei der Spezialkartierung der GK $25 \mathrm{Nr}$. 3609 Schüttdorf wurde als einziges und gleichzeitig südlichstes Vorkommen in Niedersachsen in der alten Tongrube südlich Emsbüren im oberen Teil des dort aufgeschlossenen braunen, sandigen Geschiebelehms auf $6 \mathrm{~m}$ Länge eine bis $2 \mathrm{dm}$ dicke Lage braunroten, tonigen Geschiebelehms gefunden. Die Feinkiesprobe war flintfrei, enthielt aber 8\% Old Red-Sandstein (Meyer 1988: Abb. 6, S. 36 und 51). Kuuiving et al. (1991: 2004) vergleichen dieses Vorkommen mit dem sog. „Voorst-Typ“ der ostbaltischen Moräne der Niederlande.

Die von Skupin et al. (2003:78) zitierten ostbaltischen Geschiebeinventare auf TK 25 


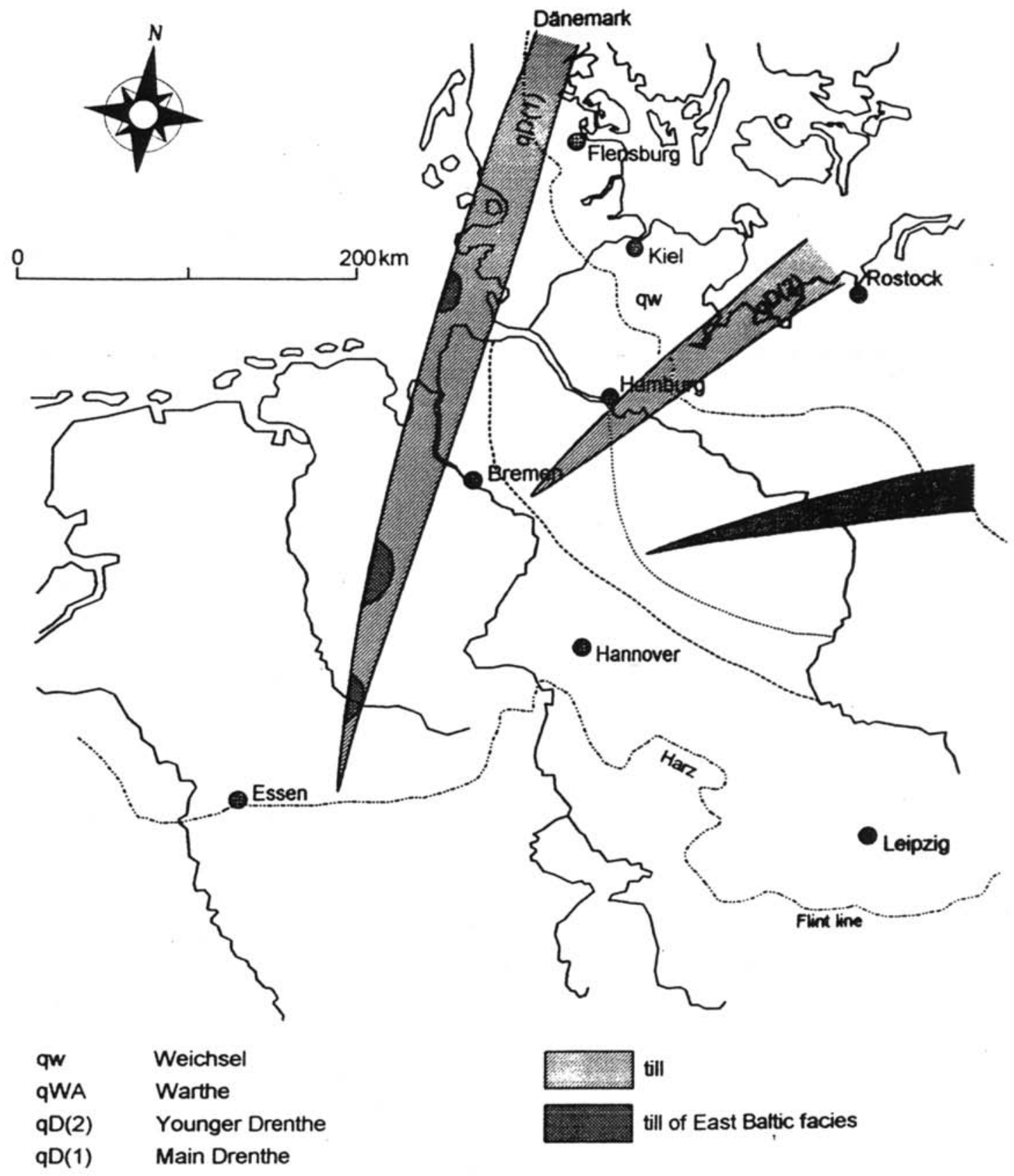

Abb. 4: Schematische Darstellung der Hauptvorstoßrichtungen und faziellen Ausbildung der SaaleGrundmoränen in Niedersachsen.

Fig. 4: Schematic ice flow directions during the Saalian and till facies in Lower Saxony. 
Nr. 3320 Liebenau stammen nicht aus roter Moräne, sondern aus Kieslagen bzw. Geschiebelehmfetzen der sog. Heisterbergphase (LÜTTIG 1958: 384). Die TGZ liegen nicht im Bereich derjenigen aus der roten Moräne, sondern randlich zu denen des Drenthe-Streufeldes. Auf dem gesamten Blatt Liebenau ist ebenso wenig wie auf den Nachbarblättern rote Moräne gefunden worden, auch nicht bei der Revisionskartierung durch den Verfasser im Jahre 1977. Dabei stellte es sich heraus, dass die auf dem ganzen Blatt weitflächig verbreitete Drenthe-Hauptmoräne auch über die Heisterberge in der SW-Blattecke hinweg zieht. Die Heisterbergphase stellt demnach keine eigenständige Phase dar, sondern ist als Teilstück der Rehburger Phase zu betrachten (Lüttig \& Meyer 1980). Eine Korrelation mit der roten Moräne kommt nicht in Frage.

Abschließend soll noch kurz auf die angebliche weitere Erstreckung der roten Drenthe-Moräne in die Altmark eingegangen werden. Weder Bohrungen noch neuere bilaterale Kartierungen der geologischen Landesämter im Grenzgebiet haben Anhaltspunkte für dortige Vorkommen ergeben. Bemerkenswert ist, dass v. PoBlozki (1995) aus einigen Gebieten der Altmark gleich hohe Dolomitgehalte in der dortigen unteren (= Drenthe) und oberen (= Warthe) Moräne erwähnt, während anderenorts der Dolomit in beiden Moränen komplett fehlt.

Als Fazit bleibt, dass im westlichen Niedersachsen nur eine Drenthe-Grundmoräne existiert, der lokal eine als Sonderfazies zu deutende ostbaltisch geprägte Moräne aufgesetzt ist. Geschiebemäßig abweichende Ausprägungen sind durch Aufnahme von Fremdmaterial oder unvollständige Durchmischung zu erklären und nicht von stratigraphischer Bedeutung.

\subsection{Jüngeres Drenthe-Stadium}

In NE-Niedersachsen, im Gebiet zwischen
Elbe, Unterweser und Aller, werden die Ablagerungen des Drenthe-Hauptvorstoßes überlagert von mächtigen und flächenhaft verbreiteten Vorschüttsedimenten eines jüngeren Vorstoßes. Ebenso weitflächig folgt darüber eine bis ca. $10 \mathrm{~m}$ mächtige Grundmoräne, die sich in der Regel durch hohen Anteil von Kreide-Kalkstein und Flint auszeichnet. Aus diesem Grund ist sie nicht selten weniger als $2 \mathrm{~m}$ tief verwittert; in diesem frischen Zustand sind graue bis hellgraue Farbtöne vorherrschend. Der Leitgeschiebeinhalt ist im Wesentlichen identisch mit dem der Hautmoräne. Nicht zuletzt aus diesem Grund wurde dieser Vorstoß in Niedersachsen dem Drenthe-Stadium zugeordnet. In Hamburg wird der äquivalente Vorstoß „Niendorfer" (Grube 1967), in Schleswig-Holstein „Kuden“-Vorstoß genannt (Stephan 1980) und dem Warthe-Stadium zugerechnet.

Diese Einbeziehung des zweiten saalezeitlichen Eisvorstoßes in das Warthestadium ist sowohl sachlich wie historisch unkorrekt. Woldstedt $(1927,1929)$ hatte als Außenrand des Warthe-Stadiums in Niedersachsen den Verlauf der Endmoränen von den Harburger Bergen über den Wilseder Berg zum Endmoränenkranz am Südrand des Uelzener Beckens erkannt, dann aber (Woldstedt 1954) unter dem Eindruck der Untersuchungen von IlliEs (1952) die Lamstedter Endmoräne (d.h. die Haupt-Eisrandlage des zweiten Saale-Vorstoßes) in das "Warthestadium im weiteren Sinne“ einbezogen. Dazu ist zu sagen, dass Illies` morphologische Studien durch keinerlei petrographische Untersuchungen gestützt waren und im Widerspruch zu den geschiebekundlichen Befunden stehen. Woldstedt (1969) hat sich neutral dahingehend geäußert, dass es zur Frage des Grenzverlaufes verschiedene Ansichten gäbe. Es ist daher auch aus Prioritätsgründen angebracht, an der ursprünglichen Auffassung WoldsTEDTs festzuhalten.

Wichtiger ist folgender Umstand: es ist bisher nicht gelungen, die Jüngere Drenthe-Grund- 
moräne wesentlich über die Landesgrenze hinaus nach Mecklenburg und Sachsen-Anhalt zu verfolgen. Es hat den Anschein, als ob diese Moräne bald mit der des älteren Vorstoßes zusammenläuft, d.h. das Rückschmelzen des Eises nicht sehr weit ging, maximal bis ins westliche Ostsee-Gebiet. Von dort kam es wieder, mit dem ausgeschürften Oberkreide-Material beladen und bevorzugt aus nord-östlicher Richtung vorstoßend, wie besonders gut im Hamburger Gebiet belegt ist (EHLers 1978). Das Jüngere Drenthe-Stadium dürfte letzten Endes nur eine, wenn auch größere Oszillation des DrentheStadiums sein; es mit dem Warthe-Stadium zu vereinen, erscheint daher unlogisch.

Unklar ist auch die Korrelation mit der saalezeitlichen Abfolge in der mitteldeutschen Typusregion. Denkbar erscheint eine Gleichsetzung der Jüngeren Drenthe mit der Leipziger Phase (EISSMANN 1975). Es ist allerdings auch darauf hinzuweisen, dass es in Brandenburg (LipPstreu 1995) Übergänge zwischen „Kreidemoräne“ und dolomitreicher Moräne gibt, was eher auf ein Zusammenlaufen der jüngeren Drenthe mit der Warthe-Moräne hinweisen würde.

Die intramoränalen Sedimente zwischen Haupt- und Jüngerer Drenthe ermöglichen keine Aussage zur Dauer und klimatischen $\mathrm{Na}$ tur zwischen beiden Vorstößen. Aus Hamburg beschrieb Grube (1967: 177) Funde von Moos und Wacholder, die auf eine Tundren-Flora weisen. In Niedersachsen sind keinerlei organogene Sedimente dazwischen gefunden worden, trotz bester Aufschlussverhältnisse, auch nicht im kontinuierlichen, gut $100 \mathrm{~km}$ langen Aufschlussprofil beim Bau des Elbe-Seitenkanals zwischen Elbe und Aller. Dabei zeigen die zahlreichen eingeschalteten Beckensedimente, dass es nicht an Sedimentfallen mangelte, in denen auch organogene Ablagerungen sich hätten bilden sollen und eine Chance zur Erhaltung gehabt hätten. Vielmehr gibt es deutliche Hinweise, dass es zumindest keine länger anhaltende Warmzeit dazwischen gegeben haben kann. Diese hätte zu einer intensiven Entkalkung der für gewöhnlich stark sandigen Haupt-Drenthe-Grundmoräne geführt. Wo diese aber durch jüngere, kalkhaltige Sedimente bedeckt und dadurch geschützt ist, fehlen in der Regel Entkalkungserscheinungen. Die außerhalb des Verbreitungsgebietes der Jüngeren Drenthe vorhandene meist totale Entkalkung der Hauptmoräne ist daher eine Folge späterer Entkalkung, d.h. vom Eem an.

\subsection{Warthe-Stadium}

Nur Nordost-Niedersachsen ist vom dritten und damit jüngsten Vorstoß der Saale-Kaltzeit erreicht worden, dem Warthe-Stadium. Hier ist deshalb die reichhaltigste Glaziärfolge entwickelt. Die guten Aufschlussverhältnisse ermöglichten seit jeher auch umfangreiche Leitgeschiebeuntersuchungen, deren Dichte in NE-Niedersachsen mit einigen 100 Zählungen die höchste ist, und die nur teilweise publiziert sind, oft nur in Tabellen oder Diagrammen (Hesemann 1939, Gauger \& Meyer 1970, Groetzner 1972, LÜtTig 1958, 2004, Lüttig \& Meyer 2002, Meyer 1998, 2000). Nach diesen Zählungen sind die glaziären Sedimente, d.h. sowohl Schmelzwasserablagerungen wie Grundmoräne, in Nordost-Niedersachsen wieder durch eine ostbaltische Geschiebegemeinschaft gekennzeichnet. Es sind die gleichen Komponenten wie in der roten Drenthe-Moräne in Südwest-Niedersachsen: viele Åland-Gesteine, hoher Anteil an grauen paläozoischen Kalksteinen und Dolomit, Old Red-Sandsteinen und sehr wenig Flint. In den Schmelzwasserkiesen sinkt durch Aufarbeitung von Drenthe-Material die baltische Komponente und steigt der Flintgehalt. Die Farbe des Geschiebemergels ist braunrot bis rotbraun, auch beim Geschiebelehm ändert sich der Farbton kaum. Der Einfachheit halber wird auch hier 
kurzerhand von „roter Moräne“ gesprochen. Im Unterschied zu den beiden vorhergehenden Vorstößen sind kaum flächenhafte Vorschüttsande entwickelt, obwohl diese stellenweise sehr mächtig werden können, am stärksten in der rückwärtigen Barendorfer Endmoräne (östlich Lüneburg), wo 10 bis $15 \mathrm{~m}$ Sand und Kies mit typisch ostbaltischer Zusammensetzung die hangende rote Warthe-Moräne von der liegenden Jüngeren Drenthe-Moräne trennen. In einem tieferen Schnitt war zeitweise auch, ebenfalls durch eine Sandschicht separiert, darunter noch die Drenthe-Hauptmoräne aufgeschlossen - einer der seltenen Fälle, wo alle drei Saale-Grundmoränen in einer Wand zu sehen waren (HöFle 1991).

Im äußeren Randgebiet, wo der WartheGletscher ausdünnte, ist wohl schon primär nur geringmächtige Grundmoräne abgelagert worden, die bevorzugt späterer Abtragung zum Opfer fiel. Erst in 20 bis $30 \mathrm{~km}$ Entfernung vom Maximalrand ist Warthe-Grundmoräne weniger lückenhaft verbreitet und können auch größere Mächtigkeiten auftreten. Maximal sind es $17 \mathrm{~m}$ in der Kartierbohrung G 17 auf GK 25 Bleckede (Meyer 2004). Wegen der sehr lückenhaften Vorschüttsedimente liegt die Warthe-Grundmoräne meist auf drenthezeitlichen Sedimenten; ihre unteren Partien sind dann stärker durch aufgenommenes Material geprägt. Wenn auch die Jüngere DrentheGrundmoräne fehlt, können auf großen Flächen drenthezeitliche Schmelzwassersedimente oberflächennah anstehen.

Für das Intervall zwischen Jüngerer Drenthe und Warthe gilt das gleiche wie für den $\mathrm{Ab}$ schnitt zwischen Haupt-Drenthe und Jüngerer Drenthe: in Niedersachsen sind keinerlei organogene Bildungen oder Böden bekannt. Aus Hamburg beschreibt GRUBE (1967) immerhin Relikte von Podsolen. Wenn Warthe-Geschiebemergel direkt auf Jüngerer Drenthe-Moräne liegt, fehlt in letzterer jede Spur von Entkalkung. Demnach ist auch hier der Zeitraum zwischen beiden Vorstößen nicht sehr lang zu bemessen, obgleich ein Eisrückschmelzen bis in den Bereich der östlichen Ostsee anzunehmen ist (Woldstedt 1954: 41).

Während des erneuten Vorrückens des Eises bis zum Außenrand Harburger Berge - Wilseder Berg etc. unterlag das Gebiet davor den periglazialen Wirkungen; u.a. wurden Hohlformen eingeebnet, auch wenn sie teilweise von Toteis plombiert waren. Aus diesem Grund ist die Zahl von Eem-Vorkommen innerhalb des Warthe-Gebietes viel größer als außerhalb: allein auf GK 25 Bleckede sind es 37 (Meyer 2004), auf dem südlichen Nachbarblatt Dahlenburg 36. Viele davon machen sich auch heute noch morphologisch bemerkbar, gelegentlich finden sich freie Wasserflächen, an Jungmoränengebiete erinnernd. Auch gibt es, wie selbst auf der quartärgeologischen Übersichtskarte 1: 500000 von Niedersachsen ersichtlich, viele kleinräumige Beckentonvorkommen und die ganze Landschaft ist lebhafter strukturiert. Wenn man also, was die Frische der Formen anbelangt, überhaupt einen Gegensatz zwischen Drenthe und Warthe sehen will, dann liegt die Grenze an der oben benannten Linie.

Die Korrelation mit den Nachbargebieten ist relativ unproblematisch: in Hamburg zum „Fuhlsbüttler Vorstoß“ (Grube 1967), in Schleswig-Holstein zum "Hennstedt-Vorstoß" (STEPHAN 1980). Unmittelbar angrenzend und in gleicher morphologischer Position ist der besonders im Raum Stendal-Gardelegen weitflächig verbreitete „Rote Altmärker Geschiebemergel“. Auch er zeichnet sich durch hohen Anteil an Åland-Kristallin, paläozoischen Kalksteinen und Dolomit aus (Hoffmann \& MeYer 1997). Weiterreichende Korrelationsversuche gestalten sich schwierig. Es bedarf noch beträchtlicher Anstrengungen, um zu einer befriedigenden Lösung zu kommen, wobei auf LeitgeschiebeZählungen nicht verzichtet werden sollte. Es ist bedauerlich, dass in den mitteldeutschen Braunkohle-Gebieten die Chance zu Leitge- 
schiebe-Studien bisher kaum genutzt wurde. Nicht zuletzt die Verschiebung der TGZ entlang des Eisrandes wäre hier in idealer Weise zu untersuchen, worauf auch LüTTIG (1999: 155) hinweist. Höchst wünschenswert wären auch entsprechende Untersuchungen in Polen, wo Einzelstudien die Brauchbarkeit der TGZ-Methode erneut bewiesen haben (BuRduKIEwICZ \& Meyer 1991, Gorska 2000, Czubla 2001). Was unter Warthe-Stadium wirklich zu verstehen ist, kann schließlich nur in der Typ-Region definiert werden und nicht in weit entfernten Randgebieten.

\section{Zur Frage einer Warmzeit zwischen}

\section{Drenthe- und Warthe-Stadium}

Vorherstehend wurde erörtert, dass in Niedersachsen innerhalb der saalezeitlichen Glaziärabfolge keinerlei organogenen Ablagerungen oder Bodenbildungen gefunden wurden, trotz guter Aufschlussverhältnisse und obwohl jeder Vorstoß eine große Anzahl von Hohlformen hinterließ, die als Sedimentfallen geeignet waren. Das trifft besonders für den HauptDrenthevorstoß zu, der beim Vorrücken im proximalen Bereich der Rehburger Stauchendmoräne stattliche Zungenbecken hinterließ, wovon das Quakenbrücker Becken im südlichen Oldenburg mit max. 30 x $15 \mathrm{~km}$ Ausdehnung das größte und tiefste ist. Aus zahlreichen Bohrungen sind hier seit langem organogene Ablagerungen bekannt, deren Altersstellung aber umstritten war, bis eine erneute Kernbohrung im Jahre 1985 Klarheit brachte (HAHNE et al. 1994). Nach dieser Bohrung liegt in 109 $\mathrm{m}$ Tiefe die Drenthe-Hauptmoräne, gefolgt von $43 \mathrm{~m}$ glazilimnischen Sedimenten, darüber das $9 \mathrm{~m}$ mächtige Eem und eine $43 \mathrm{~m}$ mächtige fluviatil-limnische Folge mit eingeschaltetem Brörup-, Odderade- und Oerel-Interstadial.
Jegliche Anzeichen für eine Warmzeit zwischen Eem und Drenthe-Hauptmoräne fehlen, obwohl die Hohlform die ideale Sedimentfalle darstellt. Das gilt gleichfalls für die anderen Abschnitte der Rehburger Phase bis in die Niederlande, und speziell dort kann schon gar nicht von einer Kenntnislücke gesprochen werden. Wenn in tiefen Hohlformen, die seit ihrer Entstehung keinerlei Erosion unterlagen, zwar reichlich eemzeitliche und jüngere, aber keine älteren organogenen Sedimente vorhanden sind, muss bezweifelt werden, dass es letztere überhaupt gab.

Auch in Nordost-Niedersachsen ist die Situation nicht anders: unabhängig von Alter und Entstehungsart der Hohlformen finden sich unter dem Eem keine älteren organogenen Ablagerungen. Bemerkenswert ist auch, dass unter dem Eem meist nicht die Warthe-Moräne folgt, sondern ältere glazigene Sedimente. Offenbar hat Drenthe-Toteis die Hohlformen plombiert und so vor der Verfüllung geschützt. Auf ähnliche Verhältnisse in Mitteldeutschland hat besonders auch EIssManN (1975:160) hingewiesen. In den nordwestdeutschen Ländern (Ehlers et al. 1984) ist man sich daher einig, dass zwischen Holstein-Dömnitz/Wacken und Eem keine weiteren Thermomere interglazialen oder interstadialen Charakters existieren. Diese zurückhaltende Bewertung beruht auch auf der Tatsache, dass die in den vergangenen Jahrzehnten postulierten Thermomere wie Ohe, Treene oder Gerdau sich sämtlich als Fehldeutungen erwiesen haben.

In den Nachbarländern ist die Situation auch nicht anders. Die unlängst wieder von Nowel (2003) aufgeführten Vorkommen angeblich intra-saalezeitlicher Warmzeiten wie Rügen oder Uecker sind teils unvollständig, teils unsicherer Position, in keinem Fall unstrittig und schon gar nicht als Referenzprofile geeignet (LITT 1994). Nicht besser ist die Situation in den östlichen Nachbarländern. Das gilt auch für den besonders interessanten, weil in der Warthe-Ty- 
pusregion gelegenen großartigen Aufschluss von Belchatow bei Lodz, wo weder der interglaziale Charakter noch die stratigraphische Position der organogenen Schichten der „Chojny-Formation" einwandfrei nachgewiesen sind, weshalb sich die Subkommission für Europäische Quartärstratigraphie bei ihrer Tagung 1994 in Lodz zu recht sehr zurückhaltend äußerte. In anderen Fällen wie bei Snaigupele (Litauen) ist der interglaziale Charakter zwar klar, nicht aber sind es die Lagerungsverhältnisse, desgl. bei Sklov, Roslavl etc., die wohl eher mit dem in den Cromer-Komplex gehörenden polnischen Ferdinandow-Interglazial $\mathrm{zu}$ vergleichen sind (ZAGWIJN 1996).

Als Fazit bleibt, dass weder in Deutschland noch in den Nachbarländern Intra-Saale-Warmzeiten (jünger als Dömnitz) nachweisbar sind und alle Fakten gegen eine solche Existenz sprechen.

\section{Ausblick}

In den letzten Jahrzehnten hat es enorme Fortschritte bei der Erforschung der „SaaleEiszeit" gegeben. Früher als mehr oder weniger einheitlich aufgefasst, allenfalls durch mehrere Rückzugsphasen gegliedert, hat sich nunmehr ein differenzierteres Bild ergeben. Der ältere Abschnitt nach dem Holstein-Interglazial, als „Untere Saale“ bezeichnet, umschließt ein bis zwei Warmzeiten mit Laubwaldentwicklung, jedoch sind zugehörige Meereshochstände bislang unbekannt, was die Korrelation mit der Tiefsee-Chronologie erschwert. Ferner sind aus den zwischengeschalteten kaltzeitlichen Abschnitten keine glaziären Sedimente nachgewiesen.

Die früher zweigeteilte glaziäre Abfolge mit Drenthe- und Warthestadium hat sich ebenfalls als vielfältiger herausgestellt mit Grundmoränen in wechselnder und sich zum Teil wiederholender Fazies und noch nicht hinreichend geklärter
Reichweite, wodurch die Korrelationen über Landes-, ja selbst Bezirksgrenzen hinaus erschwert wird. Unterschiedliche Untersuchungsmethoden sind dabei nicht gerade hilfreich, zumal Zusammenarbeit über die Ressortgrenzen eher die Ausnahme ist.

Nicht nachgewiesen sind bislang warmzeitliche Sedimente in einwandfreier Position innerhalb der saalezeitlichen Glaziärabfolge, auch nicht im benachbarten Ausland. Da ferner aus geologisch-geomorphologischen Gründen deren Existenz eher unwahrscheinlich ist, sollte bei der Aufstellung von neuen Interglazial- und Glazialzeiten Vorsicht walten.

\section{Schriftenverzeichnis}

Behrendt, L. (1998): Vergleich der Grundmoränen des Saale-Glazials der Niederlausitz mit denen Nordwestdeutschlands, der Niederlande und Polen. - Brandenburgische Geowiss. Beitr., 5 (2): 29-41, 7 Abb., 2 Tab.; Kleinmachnow.

Benda, L. (Hrsg.) (1995): Das Quartär Deutschlands. - 408 S., 95 Abb., 30 Tab.; Berlin, Stuttgart (Borntraeger).

Berg, M. W. van den \& Beets, D. J. (1987): Saalian glacial deposits and morphology in The Netherlands. - In: J.J.M. van DER MeER (ed.): Tills and Glaciotectonics: 235-251, 9 Fig.; Rotterdam (Balkema).

Burdukiewicz, J.M. \& Meyer, K.-D. (1991): The analysis of erratics from glacial deposits in Trzebnica (Silesia). - Slask. spraw. Archeol., 32: 29-42, 2Fig., 1Tab.; Wroclaw.

Ceper, A. G. (1967): Stand und Probleme der Quartärstratigraphie im Nordteil der DDR. - Ber. dt. Ges. geol. Wiss., A. Geol. Paläont., 12 (3/4): 375-404, 4 Abb., 1 Tab.; Berlin.

Czubla, P. (2001): Fennoscandian Erratics in quaternary deposits of middle Poland and their value for stratigraphic purposes. 
- (poln.; mit engl. summary). - Acta Geogr. Lodz., 80: 174 S., 53 Abb., 4 Tab.; Lodz.

EHLERS, J. (I 978): Die quartäre Morphogenese der Harburger Berge und ihrer Umgebung. - Mitteilungen der Geographischen Gesellschaft in Hamburg, 68: 181 S.; Hamburg.

EHLERS, J. (1992): Origin and distribution of red tills in North Germany. - Sverig. geol. Unders., Ca, 81: 97-105, 4Abb.; Uppsala.

Ehlers, J., Meyer, K.-D. \& Stephan, H.-J. (1984): The Pre-Weichselian Glaciations of North-West Europe. - Quat. Sci. Rev., 3: 1-40, 11 Abb., 1 Tab., 3 Taf.; Oxford.

EissmanN, L. (1975): Das Quartär der Leipziger Tieflandsbucht und angrenzender Gebiete um Saale und Elbe. - Schriftenr. f. geol. Wiss., 2: 263 S., 58 Abb., 23 Tab., 17 Taf.; Berlin.

Gauger, W. \& Meyer, K.-D. (1970): Ostbaltische Geschiebe (Dolomite, Old RedSandsteine) im Gebiet zwischen Lüneburg und Uelzen. - Der Geschiebesammler, 5: 1-12, 1 Abb., 2 Tab.; Hamburg.

GeYh, M. \& Müller, H. (2005k): Numerical $230 \mathrm{Th} / \mathrm{U}$ dating and a palynological review of the Holsteinian/Hoxnian Interglacial.Quat. Sci. Rev., 24: 1861-1872, 4 Fig., 3 Tab.; Oxford.

GorsKa, M. (2000): Some petrographical features of Vistulian lodgement till in the Central and Southern Wielkopolska Lowland and there significance towards estimating the dynamik of the last ice sheet., (pol.; mit engl. summary). - Poz. soc. art. and sci., publ. comm. Geogr. Geol., 28: 146 S., Fig., Tab; Poznan.

Groetzner, J. P. (1972): Geschiebeführung und Stratigraphie saaleeiszeitlicher Ablagerungen (Pleistozän) im Südwesten des Uelzener Beckens (Nordost-Niedersachsen). - Mit. Geol. Inst. TU Hannover, 11: 1-75, 20 Abb., 2 Taf., 7 Tab.; Hannover.

Grube, F. (1967): Die Gliederung der Saale(Riss-)Kaltzeit im Hamburger Raum. - In:
Frühe Menschheit u. Umwelt, II: 168 -195, 1Tab., 8 Taf.; Köln/Graz.

Haldorsen, S., Jörgensen, P., Rappol, M. \& Riezobos, P. H. (1989): Composition and source of the clay-sized fraction of Saalian till in The Netherlands. - Boreas, 18: 89-97., 5 Fig., 2 Tab.; Oslo.

Hahne, J., Kemle, S., Merkt, J. \& Meyer, K.-D. (1994): Eem-, weichsel- und saalezeitliche Ablagerungen der Bohrung „Quakenbrück GE 2“. - Geol. Jb., A 134: 9-69, 29 Abb., 1 Tab.; Hannover.

Hesemann, J. (1939): Diluvialstratigraphische Geschiebeuntersuchungen zwischen Elbe und Rhein. - Abh. Naturw. Verein Bremen, 31: 274-285; Bremen.

Hoffman, K. \& Meyer, K.-D. (1997): Leitgeschiebezählungen von elster- und saalezeitlichen Ablagerungen aus Sachsen, SachsenAnhalt und dem östlichen Niedersachsen. - Leipziger Geowissenschaften, 5: 115-128, 7 Abb., 3 Tab.; Leipzig.

Höfle, H.-Сh. (1991): Über die interne Struktur und die stratigraphische Stellung mehrerer Endmoränenwälle im Bereich der Nordheide bis östlich Lüneburg. - Geol. Jb., A 126: 151-169, 7 Abb.; Hannover.

Illies, H. (1952): Eisrandlagen und eiszeitliche Entwässerung in der Umgebung von Bremen. - Abh. Naturw. Ver. Bremen, 33: 19-56, 14 Abb.; Bremen.

Jerz, H. \& Linke, G. (1987): Arbeitsergebnisse der Subkommission für Europäische Quartärstratigraphie: Typusregion des Holstein-Interglazials (Berichte der SEQS 8). - Eiszeitalter u. Gegenwart, 37: 145-148, 1Abb.; Hannover.

Jöris, O. \& BAALes, M. (2003): Zur Altersstellung der Schöninger Speere. - Veröff. L.-Amt Archäologie, 57: 281-288, 4 Abb.; Halle.,

Kluiving, S., Rappol, M. \& van Der WATEREN, D. (1991): Till stratgraphy and ice movements in eastern Overijssel, The Netherlands. - Boreas, 20: 193-205, 15 Fig.; 
Oslo.

Lippstreu, L. (1995): Brandenburg. In : BendA, L. (Hrsg.): Das Quartär Deutschlands: 116 -147, 6 Abb., 2Tab.; Berlin (Borntraeger).

LitT, T. (1994): Paläoökologie, Paläobotanik und Stratigraphie des Jungquartärs im nordmitteldeutschen Tiefland. - Dissertationes Botanicae, 227: 185 S., 50 Abb., 10 Tab., 7 Beilagen; Berlin.

Litt, T. \& Turner, Ch. (1993): Arbeitsergebnisse der Subkommission für Europäische Quartärstratigraphie: Die Saalesequenz in der Typusregion (Berichte der SEQS 10). - Eiszeitalter u. Gegenwart, 43: 125 -128, 1 Tab.; Krefeld.

Lüttig, G. (1958): Methodische Fragen der Geschiebeforschung.- Geol. Jb., 75: 361418, 3 Taf., 17 Abb., 1 Tab.; Hannover.

- (1999): Geschiebestatistische Anmerkungen zur Quartärstratigraphie des nordischen Vereisungsgebietes. - Eiszeitalter u. Gegenwart, 49: 144-163, 10 Abb.; Hannover.

- (2004): Ergebnisse geschiebestatistischer Untersuchungen im Umland von Hamburg. - Arch. Geschiebekunde, 3 (8/12): 729-746, 4 Abb., 1 Tab.; Greifswald.

Lüttig, G. \& MaArleveld, G. C. (1961): Nordische Geschiebe in Ablagerungen präHolstein in den Niederlanden (Komplex von Hattem). - Geol. en Mijnb., 40: 163 -174, 6 Abb.; 's Gravenhage.

Lüttig, G. \& Meyer, K.D. (1980): Geol. Karte Niedersachsen 1 : 25 000. Erl. Blatt 3320 Liebenau: 118 S., 9 Abb., 12 Tab., 7 Kt.; Hannover.

Lüttig, G. \& Meyer, K.-D. (2002): Geschiebezählungen in der nördlichen Lüneburger Heide. - Der Geschiebesammler., 34 (4): 155-172, 2 Abb., 1 Tab.; Wankendorf.

Meyer, K.-D. (1970): Zur Geschiebeführung des Ostfriesisch - Oldenburgischen Geestrückens. - Abh. naturw. Ver. Bremen, 37 (3/2): 227-246, 4 Abb., 1 Tab.; Bremen

- (1982): Geol. Karte Niedersachsen 1 :
25 000. Erl. Blatt 2524 Buxtehude: 120 S., 22 Abb., 8 Tab., 7 Kt.; Hannover.

- (1988): Geol. Karte Niedersachsen 1 : 25000. Erl. Blatt 3609 Schüttorf: 111 S., 13 Abb., 6 Tab., 8 Kt.; Hannover.

- - (1998): Geschiebekundlich-stratigraphische Untersuchungen in der südlichen Lüneburger Heide. - Mitt. geol. Inst. Univ. Hannover, 38: 178-188, 2 Abb., 1 Tab.; Hannover.

- - (2000): Geschiebekundlich-stratigraphische Untersuchungen im Hannoverschen Wendland (Niedersachsen). - Brandenburgische Geowiss. Beitr., 7 (1/2): 115-125, 4 Abb., 1 Taf., 2 Tab.; Kleinmachnow.

- (2002): Das Emsland im Eiszeitalter. - In: Der Landkreis Emsland: 33-44, 11 Abb., 1 Tab.; Meppen.

- (2004): Geol. Karte Niedersachsen 1 : 25 000. Erl. Blatt 2730 Bleckede: 136 S., 14 Abb., 14 Tab., 3 Kt.; Hannover.

Müller, H. \& Höfle, H.-CH. (1994): Die Holstein-Interglazialvorkommen bei Bossel westlich von Stade und Wanhöden nördlich Bremerhaven. - Geol. Jb., A 134: 71-116, 13 Abb., 5 Tab.; Hannover.

Nowel, W. (2003): Zur Korrelation der Glazialabfolgen im Saale-Komplex Nord- und Mitteldeutschlands am Beispiel des Tagebaus Jänschwalde in Brandenburg. - Eiszeitalter und Gegenwart, 52: 47-83, 6 Abb., 2 Tab.; Hannover.

Poвlozki, B. v. (1995): Quaternary geology of the Altmark region. - In: Ehlers, J., KoZarski, S. \& Gibbard, PH. (ed.): Glacial Deposits in North-East Europe: 473-484, 12 Fig.; Rotterdam (Balkema).

Rappol, M. (1984): Till in Southeast Drente and the Origin of the Hondsrug Complex, The Netherlands. - Eiszeitalter u. Gegenwart, 34: 7-27, 12 Fig.; Hannover.

Rappol, M., Haldorsen, S., Jorgensen, P., Meer, J.J.M. Van der \& Stoltenberg, H.M.P. (I989): Composition and origin of 
petrographically-stratified thick till in the northern Netherlands and a Saalian glaciation model for the North Sea Basin. - Mededelingen van de Werkgroep voor Tertiaire en Kwatiaire Geologie, 26: 31-64.; Leiden.

Richter, K. (1950): Die Entwicklungsgeschichte der Täler zwischen Lathen und Verden/Aller. - Geol. Jb., 65: 641-656, 5 Abb.; Hannover.

- (1958): Geschiebegrenzen und Eisrandlagen in Niedersachsen. - Geol. Jb., 76: 223-234, 1 Taf.; Hannover.

Skupin, K., Speetzen, E. \& Zandstra, J. G. (1993): Die Eiszeit in Nordwestdeutschland. Zur Vereisungsgeschichte der Westfälischen Bucht und angrenzender Gebiete. - 143 S., 49 Abb., 24 Tab., 2 Taf., 2 Kt.; Krefeld (Geol. Landesamt).

-- (2003): Die Eiszeit in Nordost- Westfalen und angrenzenden Gebieten Niedersachsens. - 95 S., 15 Abb., 10 Tab., Anh.; Krefeld (Geol. Landesamt).

Stephan, H.-J. (1980): Glazialgeologische Untersuchungen im südlichen Geestgebiet Dithmarschens. - Schr. Naturw. Ver. Schlesw.- Holst., 50: 1-36, 17 Abb., 2 Tab., 1 Taf.; Kiel.

- (1998): Geschiebemergel als stratigraphische Leithorizonte in Schleswig-Holstein: Ein Überblick. - Meyniana, 50: 113-135, 5 Abb., 3 Tab., 1 Taf.; Kiel.

Thieme, H. (1999): Altpaläolithische Holzgeräte aus Schöningen, Lkr. Helmstedt. Bedeutsame Funde zur Kulturentwicklung des frühen Menschen. - Germania, 77 (2): 452-487, 18 Abb., Beil.; Mainz.

Urban, B. (1995): Palynological evidence of younger Middle Pleistocene Interglacials (Holsteinian, Reinsdorf and Schöningen) in the Schöningen open cast lignite mine (eastern Lower Saxony, Germany). - Meded. Rijks Geol. Dienst, 52: 175-186, 7 Fig.; Haarlem.

WATEREN, D. VAN DeR (1987): Structural geology and sedimentology of the Dammer Berge push moraine. - In: Tills and glacitectonics: 157-182, 32 Fig.; Rotterdam (Balkema).

Woldstedt, P. (1927): Über die Ausdehnung der letzten Vereisung in Norddeutschland. - Sitzungsber. preuß. geol. L.-Anst., 2: 115119; Berlin.

- (1929): Das Eiszeitalter. Grundlinien einer Geologie des Diluviums. - 406 S., 162 Abb., 15 Tab.; Stuttgart (Enke).

- (1954): Saaleeiszeit, Warthestadium und Weichseleiszeit in Norddeutschland. - Eiszeitalter und Gegenwart, 4/5: 34-48, 4 Abb.; Öhringen/Württ.

- - (1969): Quartär. Handbuch der stratigraphischen Geologie, II. - 263 S., 77 Abb., 16 Tab.; Stuttgart (Enke).

Zagwijn, W. H. (1996): The Cromerian Complex Stage of the Netherlands and correlation with other areas in Europe. - In: TuRNER, $\mathrm{CH}$. (ed.): The Early Middle Pleistocene in Europe: 145-172, 20 Fig.; Rotterdam/ Brookfield (Balkema).

Zandstra, J. G. (1976): Sedimentpetrographische Untersuchungen des Geschiebelehms von Emmerschans (Drenthe, Niederlande) mit Bemerkungen über eine Typeneinteilung der Saale-Grundmoräne. - Eiszeitalter u. Gegenwart, 27: 30-52, 7 Abb., 6 Tab.; Öhringen/Württ. 


\begin{tabular}{|l|c|c|c|}
\hline Eisqeitalter und Gegenwart & $\mathbf{5 5}$ & $\begin{array}{c}\text { 43-63 } \\
\text { 3 Abb., } 1 \text { Tab. }\end{array}$ & Hannover 2005 \\
\hline
\end{tabular}

\title{
Das Pleistozän des nördlichen Harzvorlands - eine Zusammenfassung
}

\author{
Hans-Jürgen Weymann, Ludger Feldmann \& Henning Bombien*
}

Keywords: quaternary, harz foreland, glaciofluvial/ fluvial deposits, terrace stratigraphy

Kurzfassung: Das nördliche Harzvorland liegt im Grenzbereich zwischen der ehemaligen nordischen Vereisung und dem Perigazialraum Mitteleuropas. Die ältesten pleistozänen Sedimente bilden Terrassenkiese, die vor der Elstereiszeit abgelagert wurden und einem nicht näher datierbaren Oberterrassensystem zugeordnet werden. Die Elstereiszeit ist durch Terrassenkies und glazifluviatile Sande überliefert. Die folgende Holstein-Warmzeit lässt sich nur an wenigen Stellen pedologisch nachweisen. Für den Saale-Komplex wird erstmals eine litho- und pedostratigraphische Gliederung für das Harzvorland vorgestellt. Die Fuhne-Kaltzeit und die DömnitzWarmzeit lassen sich anhand von Fließerden, Kaltzeitindikatoren sowie Böden belegen. Der darüber liegende Mittelterrassen-Kies der Saale-Kaltzeit stellt den wichtigsten Leithorizont im Pleistozän dar. Er lässt sich in mehrere Aufschüttungsphasen untergliedern. Darüber folgen stellenweise glazigene und glazifluviatile Sedimente. Die Entwässerung während der maximalen Ausdehnung des Eises erfolgte unter und vor dem Eis. Dieses wird durch die Anlage einer bedeutenden Talung sowie glazifluviatiler Ablagerungen belegt. Das Eis schmolz über mehrere Toteisphasen $\mathrm{ab}$.

In der letzten Kaltzeit herrschten im Harzvorland

\footnotetext{
*Anschriften der Verfasser: Dr. H.-J. Weymann, Wedekindstraße 12A, D-30161 Hannover, Email: h-j.weymann@freenet.de; PD Dr. habil. L. FeLDMANN, Merkurstraße 20, D-71726 Benningen, Email: ludger_feldmann@yahoo.de; Dr. H. BomBIEN, Dorfstraße 71, D-31637 Rodewald, Email: henningbombien@aol.com.
}

periglaziale Bedingungen, unter denen der Niederterrassen-Schotter in den bereits vorher existierenden Tälern abgelagert wurde.

Abstract: The northern Harz foreland is the borderland between the former northern glaciation and the periglacial area of Central Europe. The oldest sediments of the Pleistocene period are fluvial gravel deposited before the Elster glacial period. They can not be exactly dated and were assigned to a system called "Oberterrasse" (upper terrace).

The Elster glacial period is documented by fluvial gravel and melt water sand. The following Holstein interglacial period is verified only by a few pedological sites. The early Saale period can be divided for the first time in this area into periglacial sediments, ice wedges and soils. In the top of these layers follows the gravel of the "Mittelterrasse" (middle terrace). It is the most important layer of the Pleistocene in the Harz foreland. It can be divided into several accumulation periods. The middle terrace is covered by isolated glacial and periglacial sediments.

The drainage during the maximum expansion of the northern ice mass is showing by a great valley and melt water sand. The melt water eroded parts of the valleys under the ice parallel to the border of the glacier.

The retreat of the glaciers happened in form of dead ice periods.

In the last glacial period, the Weichsel period, the Harz foreland lay in the periglacial area. The valley, that was formed before this time under the ice mass, was filled with gravel of the "Niederterrasse" (lower terrace). 